\title{
Differentiating Atypical Hemangiomas and Metastatic Vertebral Lesions: The Role of T1-Weighted Dynamic Contrast-Enhanced MRI
}

\author{
(D) K.A. Morales, (D). Arevalo-Perez, DK.K. Peck, (D) A.I. Holodny, (DE. Lis, and (D) S. Karimi
}

\begin{abstract}
BACKGROUND AND PURPOSE: Vertebral hemangiomas are benign vascular lesions that are almost always incidentally found in the spine. Their classic typical hyperintense appearance on T1- and T2-weighted MR images is diagnostic. Unfortunately, not all hemangiomas have the typical appearance, and they can mimic metastases on routine MR imaging. These are generally referred to as atypical hemangiomas and can result in misdiagnosis and ultimately additional imaging, biopsy, and unnecessary costs. Our objective was to assess the utility of dynamic contrast-enhanced MR imaging perfusion in distinguishing vertebral atypical hemangiomas and malignant vertebral metastases. We hypothesized that permeability and vascular density will be increased in metastases compared with atypical hemangiomas.
\end{abstract}

MATERIALS AND METHODS: Consecutive patients from 2011 to 2015 with confirmed diagnoses of atypical hemangiomas and spinal metastases from breast and lung carcinomas with available dynamic contrast-enhanced MR imaging were analyzed. Time-intensity curves were qualitatively compared among the groups. Perfusion parameters, plasma volume, and permeability constant were quantified using an extended Tofts 2-compartment pharmacokinetic model. Statistical significance was tested using the Mann-Whitney $U$ test.

RESULTS: Qualitative inspection of dynamic contrast-enhanced MR imaging time-intensity curves demonstrated differences in signal intensity and morphology between metastases and atypical hemangiomas. Quantitative analysis of plasma volume and permeability constant perfusion parameters showed significantly higher values in metastatic lesions compared with atypical hemangiomas $(P<.001)$.

CONCLUSIONS: Our data demonstrate that plasma volume and permeability constant perfusion parameters and qualitative inspection of contrast-enhancement curves can be used to differentiate atypical hemangiomas from vertebral metastatic lesions. This work highlights the benefits of adding perfusion maps to conventional sequences to improve diagnostic accuracy.

ABBREVIATIONS: $D C E=$ dynamic contrast-enhanced; $K^{\text {trans }}=$ permeability constant; $\mathrm{VH}=$ vertebral hemangioma; $\mathrm{V}_{\mathrm{p}}=$ plasma volume

V

ertebral hemangiomas (VHs) are the most common benign tumors of the spine. They compose $28 \%$ of all skeletal hemangiomas, and the thoracic spine is the most frequent location. ${ }^{1}$ VHs are often an incidental finding, having been found in $11 \%$ of spines in a large study of postmortem examinations. ${ }^{2}$ VHs are

Received October 3, 2017; accepted after revision January 18, 2018.

From the Departments of Radiology (K.A.M., J.A.-P., K.K.P., A.I.H., E.L., S.K.) and Medical Physics (K.K.P.), Memorial Sloan Kettering Cancer Center, New York, New York; and University of Puerto Rico Medical Sciences Campus (K.A.M.), San Juan, Puerto Rico.

Krystal A. Morales and Julio Arevalo-Perez contributed equally to the work as coauthors.

This research was funded, in part, through the National Institutes of Health/National Cancer Institute Cancer Center Support Grant P30 CA008748 and the National Cancer Institute of the National Institutes of Health under award No. R25CA020449.

Paper previously presented as an electronic presentation at: Annual Meeting of the American Society of Neuroradiology and the Foundation of the ASNR Symposium, May 21-26, 2016; Washington, DC.

The content is solely the responsibility of the authors and does not necessarily represent the official views of the National Institutes of Health. more frequently found in women, especially in the fourth-tosixth decades of life. ${ }^{3}$ These lesions can affect a segment or the entirety of the vertebral body, and they are multiple in roughly one-third of patients. ${ }^{4}$ Most are stable and asymptomatic, but they can become clinically relevant with atypical imaging characteristics when establishing the presence of metastatic disease or when dealing with an aggressive/symptomatic hemangioma, which can induce pain, fractures, and neurologic manifestations due to spinal cord compression. ${ }^{5}$

Histologically, VHs are characterized by vascular spaces lined with endothelial cells of mature thin-walled blood vessels and sinuses lined by flattened or attenuated endothelium. Vessels are scattered, surrounded by a fatty matrix and vertically oriented trabeculae of bone, probably causing resorption of underlying

\footnotetext{
Please address correspondence to Sasan Karimi, MD, Department of Radiology, Memorial Sloan-Kettering Cancer Center, 1275 York Ave, New York, NY 10065; e-mail: karimis@mskcc.org

- Indicates open access to non-subscribers at www.ajnr.org

http://dx.doi.org/10.3174/ajnr.A5630
} 
bone and thickening of the remaining trabeculae. ${ }^{3}$ The characteristic radiologic appearance is defined by its histologic architecture demonstrating a parallel striated pattern, "corduroy cloth," on plain film and on sagittal or coronal CT and a spotted appearance, "polka dot," on axial CT. ${ }^{3,6}$ The MR imaging appearance is also influenced by the proportion of histologic components. Postmortem specimens of VHs have been examined with MR imaging and correlated histologically, demonstrating that the signal intensity on T1- and T2-weighted imaging is associated with the fraction of the lesion occupied by adipocytes, vessels, and edema. ${ }^{7}$ Typical VHs are hyperintense on T1 and T2, especially on FSE sequences because of their fatty stroma and vascular components, demonstrating variable enhancement, lower in lesions with a larger fatty content. ${ }^{3}$ On the other hand, atypical VHs have low fat content and high vascularity, displaying an iso-/hypointense signal on T1and hyperintense signal on T2-weighted images with elevated contrast enhancement. Also, some atypical VHs are more likely to show aggressive behavior when there is an increased vascular component and less fatty stroma. Cortical erosion, extradural soft tissue, expansion to the posterior elements, and even invasion of the spinal canal, are radiologic signs of aggressiveness. ${ }^{3}$ While these incidentally found lesions are benign, their appearance on routine STIR and T1- and T2-weighted MR images often mimics that of primary bone malignancies and metastatic lesions. ${ }^{8}$ Therefore, the ability to distinguish these commonly encountered lesions and vertebral malignancies has a direct clinical application in patient management, particularly in a patient with known malignancy.

Dynamic contrast-enhanced (DCE) MR imaging perfusion allows obtaining physiologic information about the microvascular environment of the lesion by applying a pharmacokinetic model procuring quantitative parameters such as plasma volume $\left(\mathrm{V}_{\mathrm{p}}\right)$, representing the vascular density of the lesion of interest, and permeability constant $\left(K^{\text {trans }}\right)$, which provides information about blood exchange between the vascular and interstitial compartments. These quantitative parameters have demonstrated their value in detecting the differences in the microvascular environment among metastatic lesions before and after treatment, ${ }^{9}$ benign and pathologic fractures, ${ }^{10}$ and hypovascular and hypervascular lesions. ${ }^{11,12}$ Vertebral metastases demonstrate very different histologic patterns, depending on the primary tumor. There is, however, a common feature in all of them: neoangiogenesis. The microenvironment of the metastasis induces secretion of proangiogenic growth factors that promote the development of new fragile and hyperpermeable vessels. The purpose of our study was to assess the utility of DCE MR imaging perfusion in distinguishing vertebral atypical hemangiomas and vertebral metastases, which otherwise can be undistinguishable in routine spine MR imaging. We hypothesized that permeability and vascular density would be increased in metastases because they are associated with a greater concentration of new aberrant and fragile vessels compared with hemangiomas.

\section{MATERIALS AND METHODS}

Our study was compliant with the Health Insurance Portability and Accountability Act and was approved by the institutional review board of Memorial Sloan Kettering Cancer Center. The need for informed consent was waived.

\section{Atypical VH Lesions}

A hospital data base was reviewed from January 2012 to June 2015. The query yielded 54 consecutive patients with the radiologic diagnosis of atypical VHs. The initial selection was based on the radiologic appearance (iso-/hypointensity on T1 and hyperintensity on STIR) and proved histology (if available). Cases without histology were included on the basis of the following criteria: the assessment of lesion stability after 1 year, negative PET/CT scan findings, and/or presence of the characteristic trabecular appearance on CT. Exclusion criteria from DCE MR imaging analysis comprised the following: lesions that did not meet the stability criteria after 1 year, a history of radiation therapy, or histology positive for malignancy. These selection criteria yielded 34 lesions present in 28 patients.

\section{Metastatic Lesions}

Consecutive patients who underwent spine needle biopsy during 2011-2013 were evaluated for vertebral metastatic lesions and classified on the basis of their primary carcinoma origin. Consecutive patients with primary carcinomas of breast $(n=28)$ and lung $(n=14)$ with available DCE MR imaging data were included for 42 metastatic lesions. Patients who had undergone radiation therapy, an operation, and/or kyphoplasty in the lesion of interest were excluded.

\section{Data Acquisition}

MR imaging sequences were acquired as a part of standard clinical protocol with a $1.5 \mathrm{~T}$ or $3 \mathrm{~T}$ scanner (Optima $450 \mathrm{w}$ or Discovery MR750w; GE Healthcare, Milwaukee, Wisconsin) using an 8-channel cervical-thoracic-lumbar surface coil. All patients underwent routine MR imaging, including sagittal T1weighted fast spin-echo (FOV, 32-36 cm; slice thickness, $3 \mathrm{~mm}$; TR, 400-650 ms; TE, $8.4 \mathrm{~ms}$; flip angle, $160^{\circ}$; in-plane resolution, $1.4 \times 1.4 \mathrm{~mm}^{2}$ ), T2-weighted FSE (FOV, 32-36 cm; slice thickness, $3 \mathrm{~mm}$; TR, 3500-4000 ms; TE, $110 \mathrm{~ms}$; flip angle, 160'; in-plane resolution, $1.4 \times 1.4 \mathrm{~mm}^{2}$ ), and sagittal STIR FSE (FOV, 32-36 cm; slice thickness, $3 \mathrm{~mm}$; TR, 3500-6000 ms; TE, $10 \mathrm{~ms}$; flip angle, $160^{\circ}$; in-plane resolution, $1.4 \times 1.4 \mathrm{~mm}^{2}$ ).

For DCE MR imaging, a bolus of Gd-DTPA was administered by a power injector at $0.1 \mathrm{mmol} / \mathrm{kg}$ of body weight and a rate of 2-3 $\mathrm{mL} / \mathrm{s}$. The kinetic enhancement of tissue during and after injection of Gd-DTPA was obtained before, during, and after contrast injection using a 3D T1-weighted fast-spoiled gradient recalled-echo sequence (TR, 4-5 ms; TE, 1-2 ms; slice thickness, $5 \mathrm{~mm}$; flip angle, $25^{\circ}$; FOV, $32 \mathrm{~cm}$; temporal resolution, 6-7 seconds) and consisted of 10-12 images in the sagittal plane. The duration of the DCE sequence was 200-240 seconds. Sagittal and axial T1-weighted post-Gd-DTPA MR images were acquired after DCE MR imaging.

\section{Data Processing and Analysis}

Data processing and analysis were performed using dynamic image-processing software (nordicICE; NordicNeuroLab, Bergen, Norway). Preprocessing steps integrated background noise subtraction, spatial and temporal smoothing, and detection of the arterial input function from the aorta. ROIs were manually drawn and confirmed by an experienced neuroradiologist who was blinded to the diagnosis on perfusion maps. For accurate mea- 
surement of perfusion parameters, vessels, venous structures, and vertebral body endplates were carefully excluded from the ROIs. Anatomic images that matched the DCE MR images were used in ROI placements. Time-intensity curves in the ROI were then obtained. Voxel-by-voxel estimates of quantitative perfusion parameters, including vascular permeability and plasma volume, were determined on the basis of the extended Tofts 2-compartment pharmacokinetic model. ${ }^{13}$ For lesions with multiple slices, these perfusion values were determined for each slice and the maximum values of $\mathrm{V}_{\mathrm{p}}$ and $K^{\text {trans }}$ were obtained in the ROIs and were used for statistical analysis.

\section{Imaging Analysis}

Time-intensity curve morphologies were qualitatively analyzed and compared among the groups (atypical VHs versus metastatic lesions). The time-intensity curves were classified into 5 types according to Chen et $\mathrm{al}^{14}$ : type A (a nearly flat time-intensity curve), type B (a slow-inclination curve), type C (rapid wash-in, followed by a plateau), type D (rapid wash-in followed by a washout phase), and type E (initial rapid wash-in followed by a second slowly rising phase).

For quantitative parameters, including $K^{\text {trans }}$ and $\mathrm{V}_{\mathrm{p}}$, ROI values were determined for each slice of the lesion and the maximum values of $\mathrm{V}_{\mathrm{p}}$ and $K^{\text {trans }}$ were used for the comparison.

\section{Statistical Analysis}

A Mann-Whitney $U$ test at a significance level of $P<.01$ was performed to determine the difference in the DCE MR imaging perfusion parameters, $\mathrm{V}_{\mathrm{p}}$ and $K^{\text {trans }}$, between the vertebral metastases and atypical hemangiomas.

\section{RESULTS}

A sharp difference in both morphology and signal intensity is observed when comparing time-intensity curves (Fig 1). Metastatic lesions show enhancement curves resembling type D curve morphology as described by Chen et al. ${ }^{14}$ These exhibit a sharp rise (slope) and higher peak signal intensity, followed by a faster washout phase. Most atypical hemangiomas have a minimal and delayed enhancement and fall within a narrow range, except for the 4 curves highlighted in cyan in Fig $2 A$, which deviate significantly from this range. These 4 lesions met the stability inclusion criteria and therefore were not excluded from the analysis. Although vertebral vessels were always carefully excluded from the ROIs, some of these lesions have considerable extension through the entire vertebral body and could represent rare cases in which atypical hemangiomas display an aggressive behavior and substantial vascularity and vertebral body invasion occur. If we evaluated the curve average intensity excluding these 4 cases (Fig 2B), $88 \%$ of the atypical hemangioma curves analyzed would show a characteristic pattern with minimum and late enhancement curves with no evident washout in contrast to metastases, which display a rapid wash-in phase, higher peak, and clear washout (Fig 2C).

Quantitative analysis demonstrates higher perfusion values $\left(\mathrm{V}_{\mathrm{p}}\right.$ and $\left.K^{\mathrm{trans}}\right)$ of metastatic lesions than atypical hemangiomas $(P<.01)$, despite an almost identical appearance on T1 (Figs 3 and 4$). V_{p}$ values demonstrated the greatest difference and mini- mal overlap between atypical vertebral hemangiomas and metastatic lesions, being the best biomarker. No significant differences were found when comparing lung and breast metastases $(P>.3)$.

\section{DISCUSSION}

We compared 34 atypical hemangiomas and 42 metastatic vertebral lesions and found that DCE MR perfusion assessment of the TI curves and $\mathrm{V}_{\mathrm{p}}$ and $K^{\text {trans }}$ parameters demonstrated higher values in vertebral metastases compared with atypical vertebral hemangiomas. $V_{p}$ was considered the best parameter to differentiate the 2 groups.

Noninvasive imaging biomarkers can be of paramount importance to help distinguish atypical VHs and metastatic vertebral lesions, especially when atypical VHs can mimic the pattern observed for primary malignant and metastatic lesions. Currently, long-term stability and the classic radiologic appearance are the imaging tools available to confirm the diagnosis. However, the vertical trabecular appearance has been reported to be more difficult to visualize in atypical hemangiomas, making the diagnosis even more challenging. ${ }^{8}$ Furthermore, although rare, VHs display "aggressive" behavior that can manifest as pain and radiculopathies when involvement of the entire vertebral body and compression of the spinal cord and nerve roots occur. These can be easily mistaken for malignancies and may result in additional diagnostic imaging, biopsy, and, in some cases, an operation. In addition, atypical VHs have been identified concurrent with metastatic disease from primary malignancy such as colon adenocarcinoma ${ }^{15}$ and thyroid cancer. ${ }^{16}$ Consequently, their accurate identification in these cases poses a major challenge with a large impact on patient staging and management.

In the past decade, our tools to identify these lesions have been limited. Effort has been made to differentiate hemangiomas and malignant lesions with the use of diffusion-weighted imaging and apparent diffusion coefficient calculations. ${ }^{17}$ Quantitative chemical shift MR imaging has also been used, and a proportional change in signal intensity on in-phase compared with out-ofphase images showed a decrease in signal intensity in benign lesions compared with metastatic lesions. ${ }^{18}$ However, no robust method has been established to differentiate atypical hemangiomas and metastatic spine lesions.

Our study takes advantage of spine DCE MR imaging perfusion as a noninvasive method of obtaining quantitative parameters representing the physiology of the microvascular environment of each lesion. Except for the aforementioned 4 cases, atypical hemangioma time-intensity curves had a minimal and delayed enhancement, with no evident washout in contrast to metastases, which display a rapid wash-in phase, higher peak, and clear washout, a common indicator of malignancy. In addition to the benign appearance of the curves, quantitative analysis of the perfusion parameters $\mathrm{V}_{\mathrm{p}}$ and $K^{\text {trans }}$ also demonstrated significantly lower perfusion values in atypical hemangiomas compared with metastatic lesions, despite an almost identical appearance on T1-weighted sequences.

Our findings reflect the physiology and histologic structure of atypical vertebral hemangiomas, less fat and greater vascular content. Most hemangiomas in our study showed low plasma volume and permeability. Only 4 cases demonstrated higher $V_{p}$ values, 
Atypical Hemangiomas
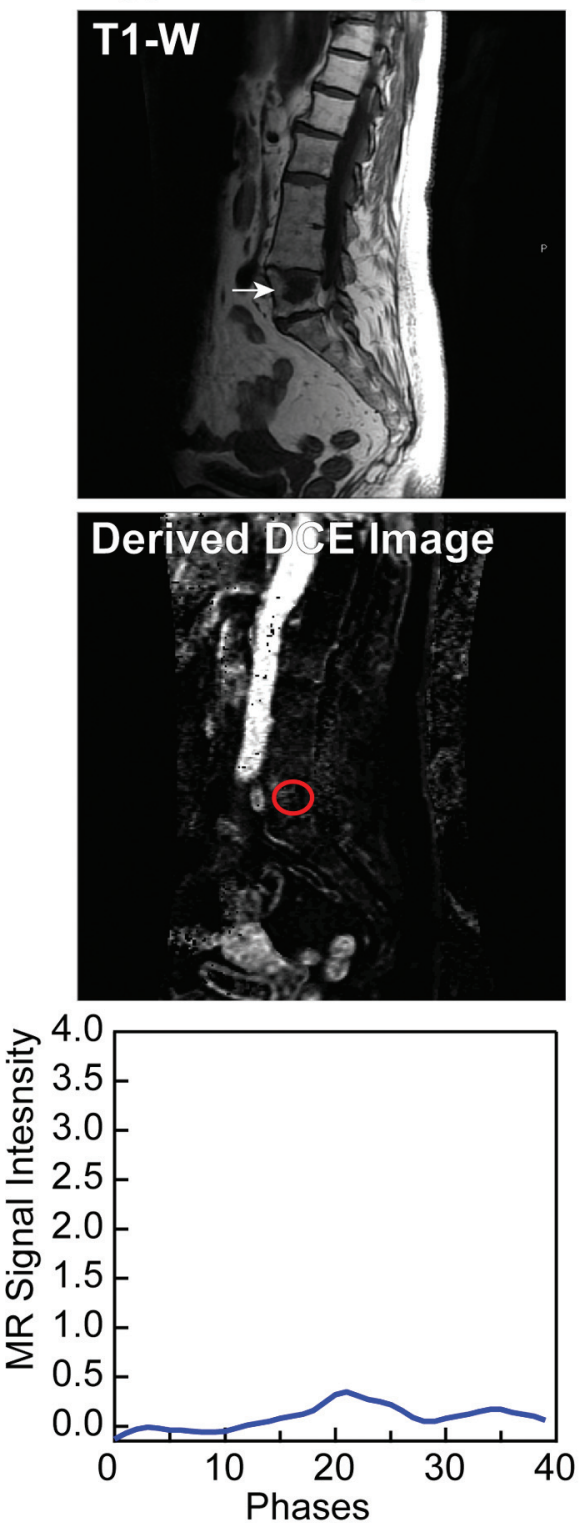

Breast
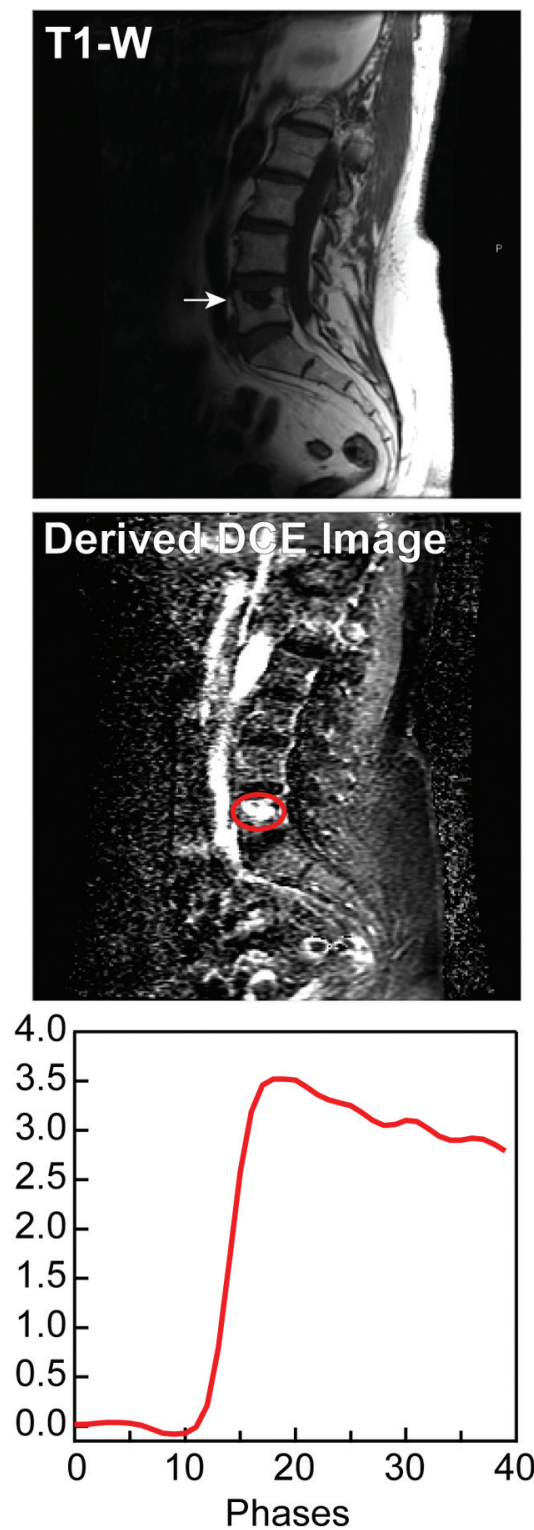

Lung
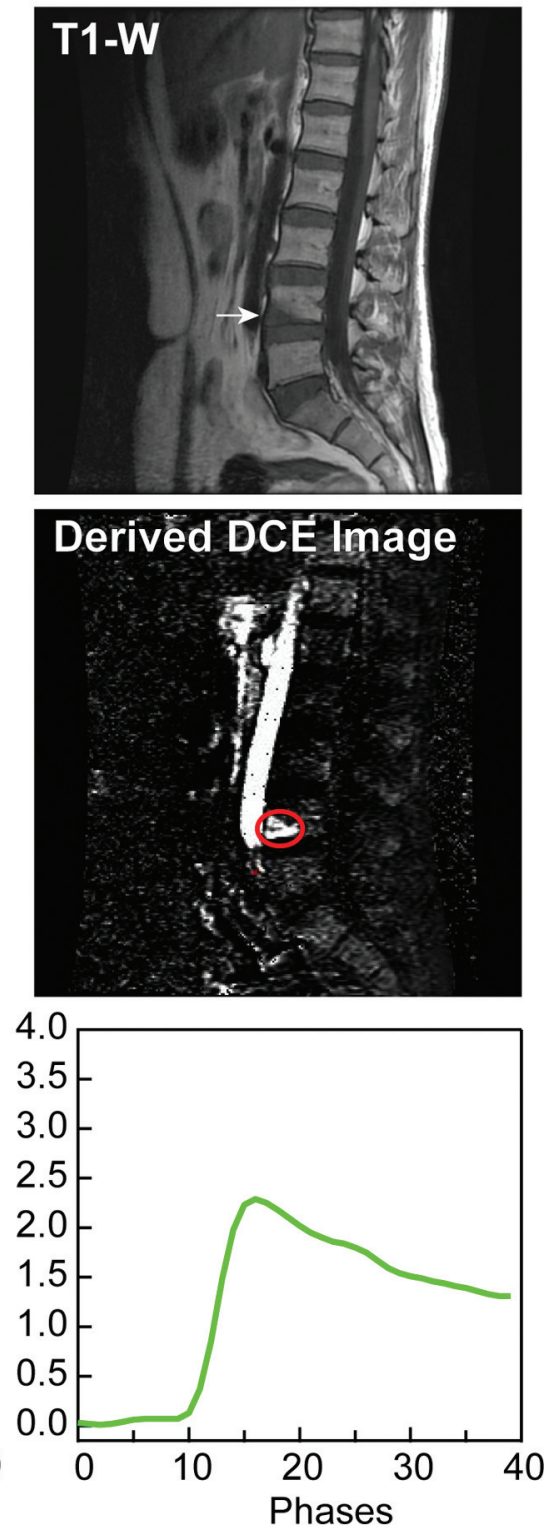

FIG 1. Representative sagittal T7-weighted and dynamic images derived from DCE MR imaging for atypical hemangiomas and spinal lesions originating from primary breast and lung carcinomas. The lower panel corresponds to the MR imaging signal enhancement curve as a function of phases (time) obtained for each representative lesion shown.
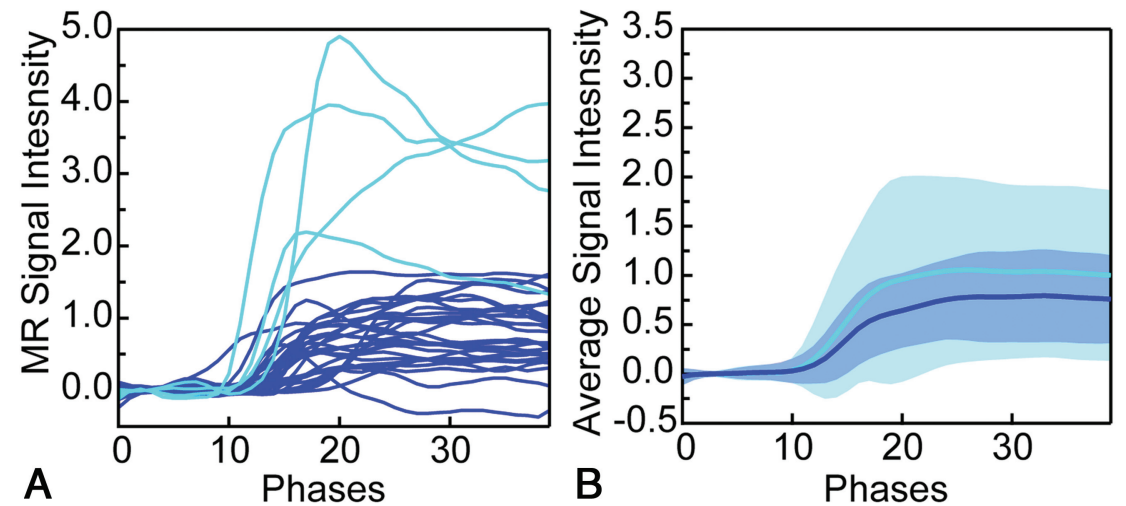

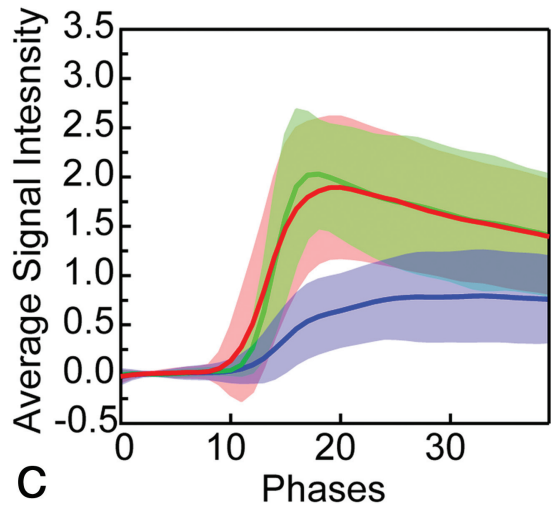

FIG 2. $A$, Enhancement curve for all 34 atypical hemangiomas. Curves highlighted in cyan show the 4 cases with elevated enhancement. $B$, Average enhancement curves for all atypical hemangiomas (cyan) excluding atypical hemangiomas with elevated enhancement (blue). C, Average enhancement curves for all atypical hemangiomas excluding enhancement curve outliers (blue) compared with breast (red) and lung (green) metastases. 


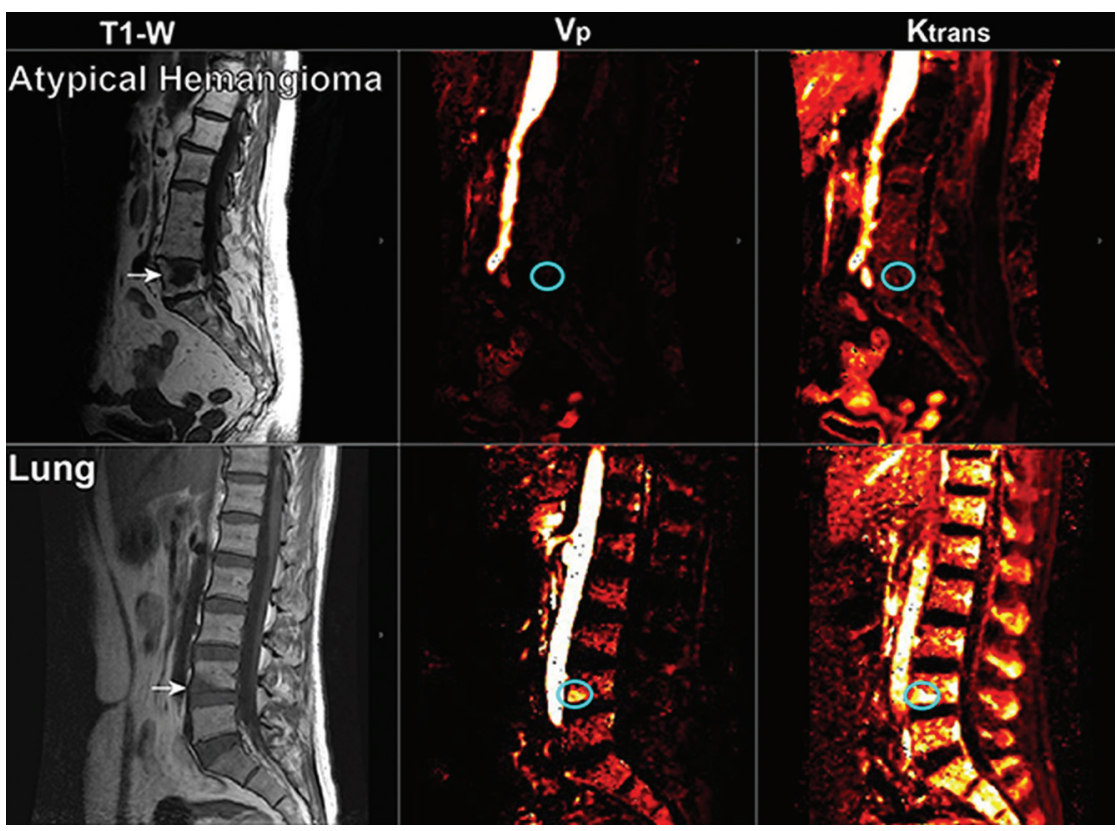

FIG 3. Representative sagittal T1-weighted imaging and the corresponding perfusion maps for $V_{p}$ and $K^{\text {trans }}$ parameters for atypical hemangiomas and metastasis from lung carcinoma. Arrows indicate the level of the lesion in $\mathrm{TIWI}$, and the cyan circle highlights the region on $\mathrm{V}_{\mathrm{p}}$ and $K^{\text {trans }}$ maps where the lesion is located.
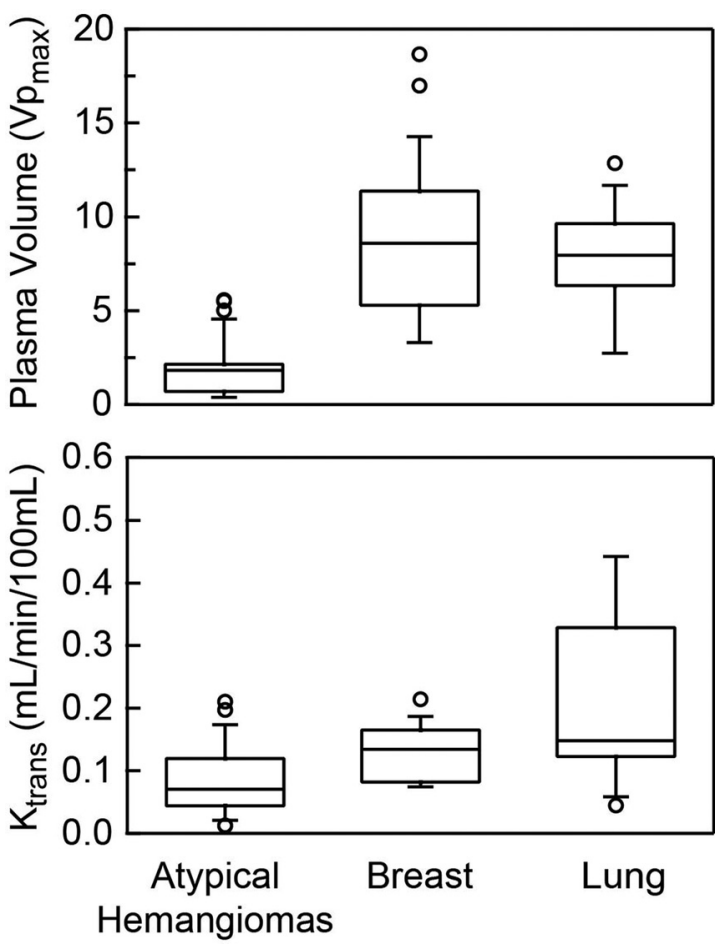

FIG 4. $V_{p}$ and $K^{\text {trans }}$ parameters obtained for atypical hemangiomas and metastatic lesions from breast and lung carcinoma. Metastases show significantly higher values $(P<.05)$ for both $\mathrm{V}_{\mathrm{p}}$ and $K^{\text {trans }} \mathrm{com}-$ pared with atypical vertebral hemangiomas. Note that the 4 outlier values representing aggressive hemangiomas account for the higher $V_{p}$ values in the atypical hemangioma group that demonstrate minimal overlap with the metastatic group and remain low compared with other metastases. which overlap those of the metastatic lesions. These 4 cases probably represent aggressive or compressive vertebral hemangiomas, which exhibit a higher degree of vascularization and an expansile soft-tissue component. ${ }^{19}$ This subgroup is associated with some features that help distinguish it from atypical quiescent hemangiomas, such as involvement of the entire vertebral body, extension into the neural arch, cortical expansion, midthoracic location, irregular honeycomb pattern, and soft-tissue mass, according to Laredo et al. ${ }^{19}$ In fact, 3 of the 4 lesions were expanding into the posterior vertebral elements, and one demonstrated ventral epidural disease. The remaining lesion was a small well-demarcated hemangioma confined to the vertebral body. However, the 4 cases demonstrated lower values of $K^{\text {trans }}(0.08,0.08,0.09$, and 0.11$)$ than those seen on metastases $\left(K^{\text {trans }}\right.$ mean, 0.15 ), in keeping with the expected low permeability due to a theoretically preserved endothelium in these lesions.

As with Laredo et al, ${ }^{19}$ we suggest that DCE MR imaging perfusion can be a useful tool for differentiating quiescent atypical VHs that represent localized, relatively inactive angiomatous dystrophies from aggressive atypical VHs that show extensive, active angiomatous tumors that can compress the spinal cord and become symptomatic. ${ }^{19}$ When there is an abnormally elevated $V_{p}$, $K^{\text {trans }}$ can be helpful in demonstrating preservation of permeability, suggesting a benign etiology.

Our study has several limitations. First, it is a retrospective study with the possibility of selection bias. Second, there was no pathologic confirmation for most of the atypical hemangiomas; however, all these lesions were stable after 1 year, had negative findings on PET/CT, and/or had the characteristic trabecular appearance on CT. Third, the manual ROI selection, though a standard method of analyzing perfusion data, is an operator-dependent technique associated with potential interobserver and intraobserver variability. However, having 1 single trained operator performing the measurements limits the potential variation. Fourth, there is limited representation of other malignancies in the metastatic group that could have demonstrated different perfusion patterns. On the other hand, lung and breast are by far the most representative primary malignancies, accounting for $31 \%$ and $24 \%$ of all sources of spinal metastatic disease. Despite these limitations, this is the first study of its kind to compare atypical VHs and vertebral metastases using DCE MR imaging, to our knowledge.

\section{CONCLUSIONS}

Our data demonstrate that qualitative inspection of contrast-enhancement curves and quantitative analysis of $\mathrm{V}_{\mathrm{p}}$ and $K^{\text {trans }}$ perfusion parameters can be used to differentiate atypical hemangi- 
omas from vertebral metastatic lesions. The ability of DCE MR imaging to noninvasively characterize tumor vascularity has important clinical implications, which could lead to changes in patient management, reducing biopsies, hospital stay, additional imaging, and patient anxiety.

Disclosures: Krystal A. Morales—RELATED: Grant: NIH/NCI Cancer Center Support Grant P30 CA008748, National Cancer Institute R25CA020449, Comments: Medical Student Summer Fellowship Program of the Memorial Sloan Kettering Cancer Center is a research program for first- and second-year students at US medical schools who are interested in careers as physician-scientists in the field of oncology and/or related biomedical sciences. Funded since 1977 by the National Cancer Institute, this program grants students the opportunity to conduct basic laboratory or clinical research under the mentorship of Memorial Sloan Kettering faculty. Julio ArevaloPerez-RELATED: Grant: NIH/NCI Cancer Center Support Grant P30 CA008748.* K.K. Peck-RELATED: Grant: NIH/NCI Cancer Center Support Grant P30 CA008748. A.I. Holodny-RELATED: Grant: NIH/NCl Cancer Center Support Grant P30 CA008748. Eric Lis-RELATED: Grant: NIH/NCI Cancer Center Support Grant P30 CA008748; UNRELATED: Payment for Lectures Including Service on Speakers Bureaus: Medtronic. S. Karimi-RELATED: Grant: NIH/NCI Cancer Center Support Grant P30 CA008748. *Money paid to the institution.

\section{REFERENCES}

1. Murphey MD, Fairbairn KJ, Parman LM, et al. From the archives of the AFIP: musculoskeletal angiomatous lesions: radiologic-pathologic correlation. Radiographics 1995;15:893-917 CrossRef Medline

2. Junghanns H, Schmorl G. The Human Spine in Health and Disease. New York: Grune \& Stratton; 1971

3. McEvoy SH, Farrell M, Brett F, et al. Haemangioma, an uncommon cause of an extradural or intradural extramedullary mass: case series with radiological pathological correlation. Insights Imaging 2016;7:87-98 CrossRef Medline

4. Karlin CA, Brower AC. Multiple primary hemangiomas of bone. AJR Am J Roentgenol 1977;129:162-64 CrossRef Medline

5. Alexander J, Meir A, Vrodos N, et al. Vertebral hemangioma: an important differential in the evaluation of locally aggressive spinal lesions. Spine (Phila Pa 1976) 2010;35:E917-20 CrossRef Medline

6. Persaud T. The polka-dot sign. Radiology 2008;246:980-81 CrossRef Medline

7. Baudrez V, Galant C, Vande Berg BC. Benign vertebral hemangioma: MR-histological correlation. Skeletal Radiol 2001;30:442-46 CrossRef Medline

8. Gaudino S, Martucci M, Colantonio R, et al. A systematic approach to vertebral hemangioma. Skeletal Radiol 2015;44:25-36 CrossRef Medline

9. Chu S, Karimi S, Peck KK, et al. Measurement of blood perfusion in spinal metastases with dynamic contrast-enhanced magnetic resonance imaging: evaluation of tumor response to radiation therapy. Spine (Phila Pa 1976) 2013;38:E1418-24 CrossRef Medline

10. Arevalo-Perez J, Peck KK, Lyo JK, et al. Differentiating benign from malignant vertebral fractures using T1-weighted dynamic contrastenhanced MRI. J Magn Reson Imaging 2015;42:1039-47 CrossRef Medline

11. Khadem NR, Karimi S, Peck KK, et al. Characterizing hypervascular and hypovascular metastases and normal bone marrow of the spine using dynamic contrast-enhanced MR imaging. AJNR Am J Neuroradiol 2012;33:2178-85 CrossRef Medline

12. Saha A, Peck KK, Lis E, et al. Magnetic resonance perfusion characteristics of hypervascular renal and hypovascular prostate spinal metastases: clinical utilities and implications. Spine (Phila Pa 1976) 2014;39:E1433-40 CrossRef Medline

13. Tofts PS, Brix G, Buckley DL, et al. Estimating kinetic parameters from dynamic contrast-enhanced T(1)-weighted MRI of a diffusable tracer: standardized quantities and symbols. J Magn Reson Imaging 1999;10:223-32 CrossRef Medline

14. Chen WT, Shih TT, Chen RC, et al. Blood perfusion of vertebral lesions evaluated with gadolinium-enhanced dynamic MRI: in comparison with compression fracture and metastasis. J Magn Reson Imaging 2002;15:308-14 CrossRef Medline

15. Zapalowicz K, Bierzyńska-Macyszyn G, Stasiów B, et al. Vertebral hemangioma coincident with metastasis of colon adenocarcinoma. J Neurosurg Spine 2016;24:506-09 CrossRef Medline

16. Laguna R, Silva F, Vazquez-Sellés J, et al. Vertebral hemangioma mimicking a metastatic bone lesion in well-differentiated thyroid carcinoma. Clin Nucl Med 2000;25:611-13 CrossRef Medline

17. Leeds NE, Kumar AJ, Zhou XJ, et al. Magnetic resonance imaging of benign spinal lesions simulating metastasis: role of diffusionweighted imaging. Top Magn Reson Imaging 2000;11:224-34 CrossRef Medline

18. Zajick DC Jr, Morrison WB, Schweitzer ME, et al. Benign and malignant processes: normal values and differentiation with chemical shift MR imaging in vertebral marrow. Radiology 2005;237:590-96 CrossRef Medline

19. Laredo JD, Reizine D, Bard M, et al. Vertebral hemangiomas: radiologic evaluation. Radiology 1986;161:183-89 CrossRef Medline 